\title{
Metastatic Clear Cell Renal Cell Carcinoma: The Great Pretender and the Great Dilemma
}

\author{
Umberto Maestroni $^{\mathrm{a}}$, Donatello Gasparro ${ }^{\mathrm{b}}$, Francesco Zigliolia, \\ Giulio Guarino $^{\text {a }}$, Davide Campobasso ${ }^{\text {a, c }}$ (1)
}

\begin{abstract}
Metastatic renal cell carcinoma ( $\mathrm{mRCC}$ ) may present with a wide range of clinical pictures. Reportedly, paraneoplastic syndromes are the first sign in $20 \%$ of cases and only $15 \%$ of cases show the classic triad (flank pain, gross hematuria, and palpable abdominal mass) at presentation. The remaining cases present with signs and symptoms related to the site of distant metastases. These data may explain the reason why about $20-30 \%$ of patients are metastatic at presentation. We report the case of a 63-year-old woman who came to our attention for lower back pain. After imaging studies, we detected a left kidney mass of $86 \times 61 \times 79$ $\mathrm{mm}$, multiple right pulmonary nodules and six bone lesions. She underwent left radical nephrectomy. After 1 month, she developed signs of spinal cord compression with neurological deficits and she underwent emergency spinal decompression. In order to allow complete motor recovery, the subsequent stereotactic body radiation therapy was not performed, and she is currently taking combination immunotherapy regimens. Management of $\mathrm{mRCC}$ is in a continuous evolution due to availability of new target therapies and the possibility of a multimodal approach with surgical, focal and radiotherapy treatments. However, the ideal treatment algorithm is yet to come. This is why mRCC diagnosis and management are still challenging for the clinicians.
\end{abstract}

Keywords: Metastatic renal cell carcinoma; Diagnosis; Cytoreductive nephrectomy; Immunotherapy-based combination therapy; Multidisciplinary management

\section{Introduction}

Renal cell carcinoma (RCC) accounts for around 3\% of all solid cancers, with an increasing incidence in the last years

Manuscript submitted August 13, 2021, accepted September 30, 2021

Published online October 21, 2021

aDepartment of Urology, University Hospital of Parma/Azienda OspedalieroUniversitaria di Parma, Via Gramsci 14, Parma 43126, Italy

bMedical Oncology Unit, University Hospital of Parma/Azienda OspedalieroUniversitaria di Parma, Via Gramsci 14, Parma 43126, Italy

${ }^{\mathrm{c} C}$ Corresponding Author: Davide Campobasso, Department of Urology, University Hospital of Parma/Azienda Ospedaliero-Universitaria di Parma, Via Gramsci 14, Parma 43126, Italy. Email: d.campobasso@virgilio.it

doi: https://doi.org/10.14740/wjon1406 and the highest incidence in Western countries [1]. RCC encompasses different histologic subtypes. The most common subtypes are clear cell RCC (ccRCC), papillary RCC (pRCC) and chromophobe RCC (chrRCC), representing 75-85\%, 10$15 \%$ and $5-10 \%$ of all histological categories of kidney cancers, respectively [2]. As these tumors arise from retroperitoneum, signs and symptoms of presentation are related to local invasion of surrounding tissues and organs as well as to the site of metastasis. This explains the reason why about $20-30 \%$ of patients are metastatic at presentation. It is well known that metastatic RCC (mRCC) is a poor prognosis disease, with a 5 -year survival rate of $8 \%$ only [3]. However, in the last decade, the refinements in systemic therapies of $\mathrm{mRCC}$, with the introduction of the antiangiogenic tyrosine kinase inhibitors (TKIs) and the immune checkpoint inhibitors (CKIs), have prolonged the overall survival (OS) [4]. Due to the more and more widespread use of ultrasound imaging for the evaluation of a variety of nonspecific symptoms, more than $50 \%$ of renal masses are detected incidentally [5]. As a consequence, paraneoplastic syndromes are the first sign in $20 \%$ of cases. In addition, $10 \%$ of patients seek medical attention for hematuria and flank pain. In the remaining cases, the presenting signs and symptoms are attributable to distant metastases [6], with the most common sites including lung, lymph nodes, bone, and liver. We report on a case of a patient with oligometastatic RCC whose first symptom was back pain, and she was treated with upfront cytoreductive nephrectomy $(\mathrm{CN})$ and a combination of immunotherapy regimens.

\section{Case Report}

A 63-year-old female patient came to our attention for lower back pain. Her past medical history was significant for hypertension and pulmonary sarcoidosis treated with steroids. Neurological examination revealed a lumbar spinal syndrome without motor deficit. After failure of medical management, spinal computed tomography (CT) scan was performed, demonstrating a spinal lesion of $24 \times 23 \mathrm{~mm}$ at the seventh thoracic vertebra (Fig. 1). A thoraco-abdomen CT scan revealed an 86 $\times 61 \times 79 \mathrm{~mm}$ mass at the left kidney (Fig. 2) with multiple right pulmonary nodules and six bone lesions confirmed at the bone scan (Fig. 3). At diagnosis, Karnofsky Performance Status (KPS) was 80. The International Metastatic RCC Database Consortium (IMDC) risk score was intermediate and the Memorial Sloan-Kettering Cancer Center (MSKCC) score was 


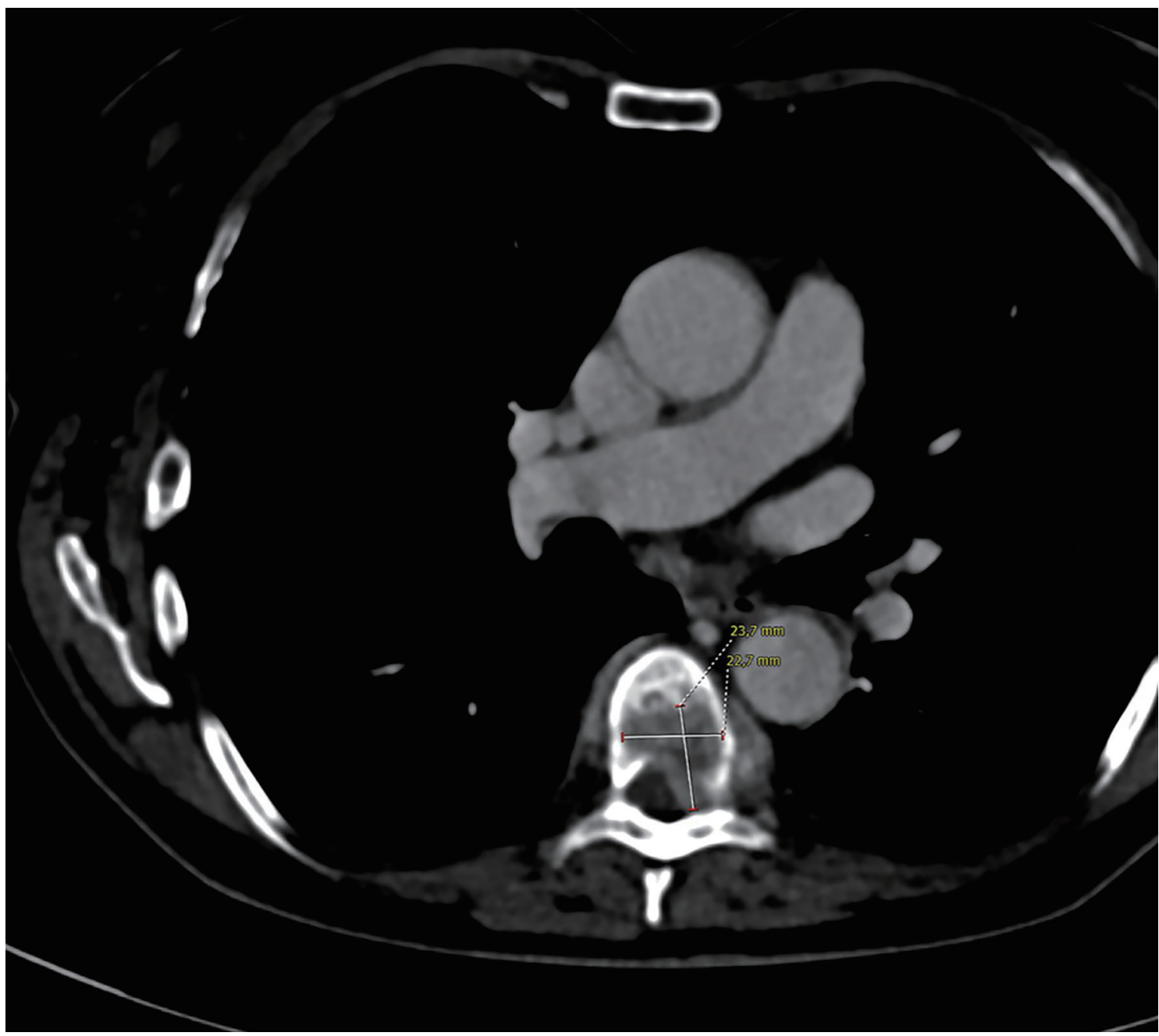

Figure 1. Lumbar computed tomography scan revealing a spinal lesion of $24 \times 23 \mathrm{~mm}$ at the seventh thoracic vertebra.

intermediate. After multidisciplinary discussion, the patient underwent open trans-peritoneal left radical nephrectomy. The histology results described a ccRCC grade 2 with tumor ex- tension in segmental (muscle-containing) venal branches and perirenal fat with clear margins (pathological T3aR0Nx). One month after surgery, she developed signs of spinal cord com-

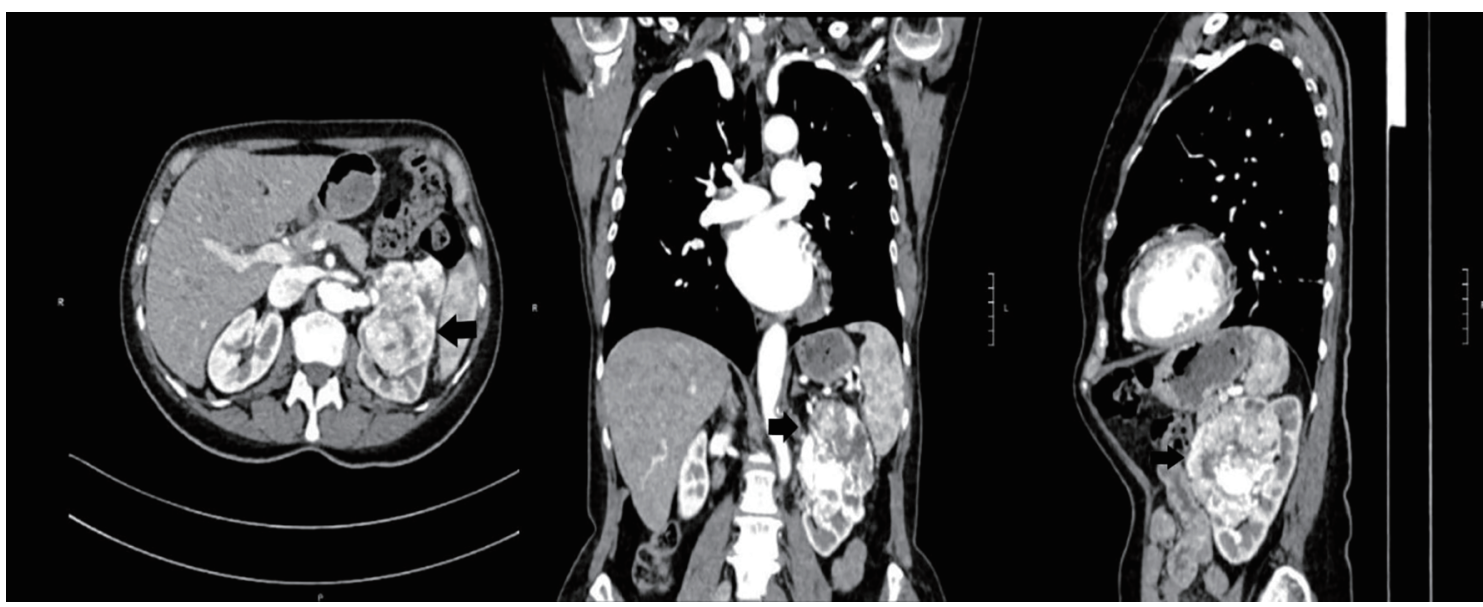

Figure 2. Abdominal computed tomography scan. Arrow indicates a left renal cell carcinoma. 

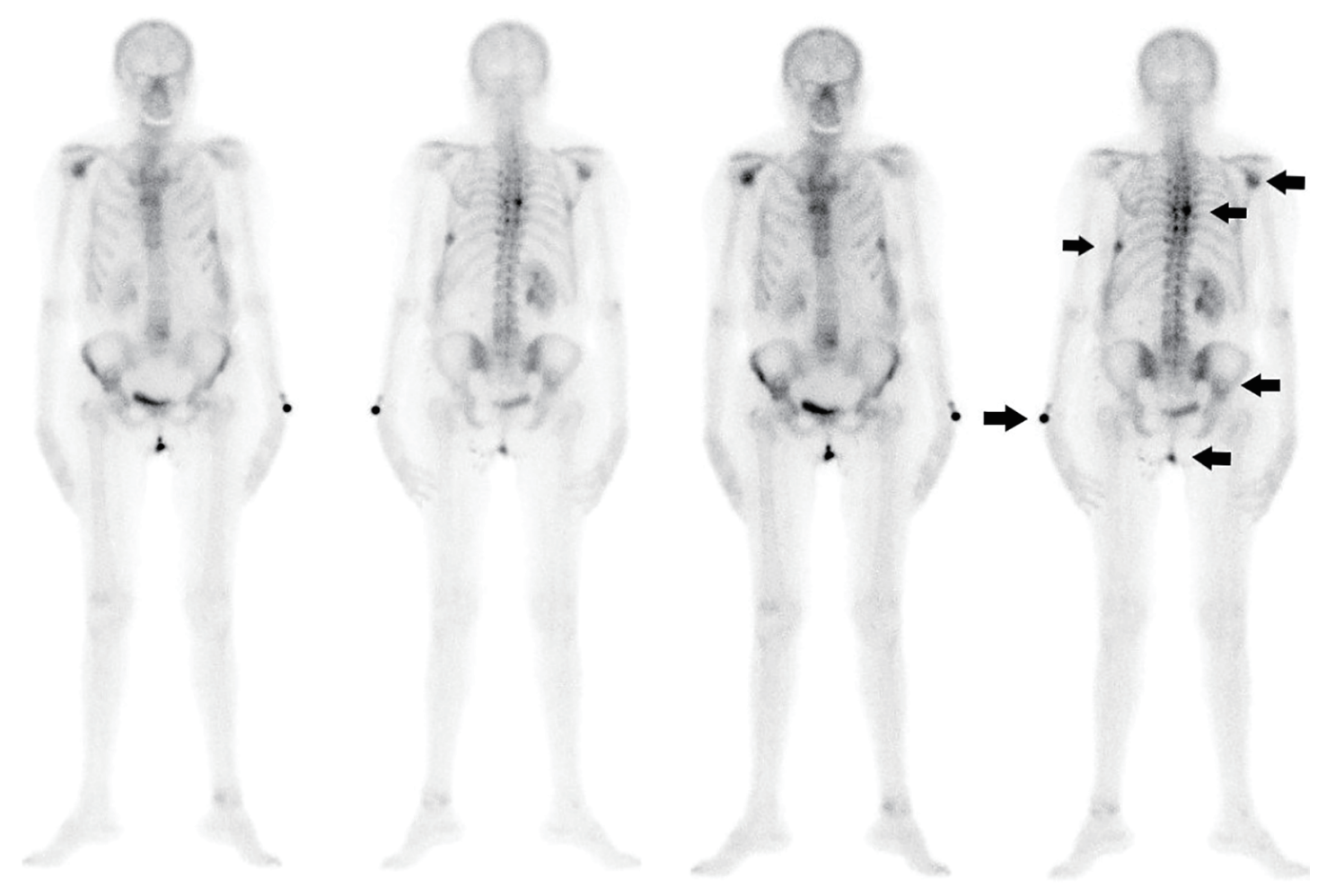

Figure 3. The $99 \mathrm{mTc}$ bone scan confirmed six bone metastases (arrows).

pression with neurological deficits and underwent emergency spinal decompression with a D7 laminectomy and stabilization of D5/D9. After neurosurgical operation and physiotherapy, she achieved complete remission of back pain and neurological deficits. A radiotherapy treatment of the vertebral metastases after surgery was avoided in order not to jeopardize complete functional recovery. The follow-up thoraco-abdomen CT scan after 3 months ruled out disease progression. She is currently on an immunotherapy regimen with avelumab (anti-programmed cell death-1 ligand, programmed death ligand 1) and axitinib (a vascular endothelial growth factor receptor TKI).

\section{Discussion}

After the introduction of the ultrasonography (US), which is cost-effective and no-invasive, the number of patients diagnosed with renal cancer has increased. Nowadays, we can maintain that about $50 \%$ of renal cancers are detected while investigating other symptoms. This led to the early detection of renal masses, making it unusual the finding of the classical triad made up of flank pain, macrohematuria and palpable abdominal mass. Nevertheless, in $20 \%$ of cases, patients present with paraneoplastic syndromes. Among these, the most common are hypercalcemia, hypertension, polycythemia and Stauffer's syndrome [6]. In the remaining cases, renal cancer is found while investigating the symptom(s) of presentation. This is what happened in our case. In this view, it is not surpris- ing that RCC has been defined "the great imitator". Persistent bone pain not responding to medical therapy needs further investigation to confirm, or rule out, the skeleto-muscular origin. In our case, spinal magnetic resonance imaging performed in the hypothesis of a lumbar discal herniation or osteoporotic vertebral burst fracture actually revealed the presence of the left kidney mass with metastases.

Noteworthy, the management of mRCC is not less difficult than its initial diagnosis. The range of treatment strategies of mRCC is ever evolving, due to new medical and target therapies. The most appropriate treatment has to be tailored on disease characteristics and patients' comorbidities. If radical nephrectomy has a crucial role for local disease control in case of hematuria and flank pain, its role in metastatic disease is still debated. Some evidence supports $\mathrm{CN}$ in selected patients, in association with new target therapies, thus making $\mathrm{CN}$ still relevant [7]. Last but not least, $\mathrm{CN}$ provides a histological diagnosis that may be of help in defining the following treatment strategy. Conversely, in case of poor performance status, a percutaneous CT- or US-guided renal biopsy is appropriate in order to obtain a histological diagnosis with low risk of major complications and it can be helpful to guide the target therapies' choice [8]. In these cases of mRCC who require aggressive surgery that cannot be safely offered due to the poor performance status, as reported by some authors, targeted therapies before surgery can play a cytoreductive effect on the primary tumor, making the nephrectomy feasible [9].

In our case, after multidisciplinary consultation, we de- 
cided to perform a radical nephrectomy taking into account patient's age, good performance status and the risk category. One month after surgery, the patient showed the signs of spinal cord compression, which required surgical decompression. Then she was started on an immunotherapy-based combination therapy, which in the literature is reported to be superior to standard first-line target therapy [10]. An additional consideration when managing $\mathrm{mRCC}$ is the importance of a multidisciplinary team. In the therapeutic armamentarium for $\mathrm{mRCC}, \mathrm{CN}$ and local treatment of metastases play an ambiguous role [11]. Despite their role in oligometastatic patients, with good-to-moderate performance status, correct timing and patients' selection are still not clear. The possibility to perform various systemic and local treatments has increased the opportunity to effectively manage the patients with $\mathrm{mRCC}$ [12]. In this view, a close cooperation between the pathologist, the radiologist, the radiotherapist, the urologist, the oncologist as well as other surgical specialists is the key to offer the best chance of prolonging OS. The management of bone metastasis highlights the importance of the multidisciplinary discussion. On one side, some retrospective evidence favors stereotactic body radiation therapy (SBRT) for secondary lesions, based on its safety and the opportunity to improve the local disease control and the potential synergy with immunotherapy regimens [13]. On the other side, in the specific case of vertebral metastases, surgical treatment is a consolidated procedure. However, in our case, after spinal surgery we decided not to perform a post-operative SBRT in order to favor a complete neurological recovery [14], which was considered a priority in the management of this case. This choice came from considering the histological type and from balancing treatment benefits and risks.

Unfortunately, there is a lack of clinical, histological and genetic data in the literature and more studies are necessary to reach a consensus on precise and validated algorithms for stepwise treatment of metastases in patients with good performance status. An algorithm for the best treatment of $\mathrm{mRCC}$ is still far from being available $[15,16]$. As we reported in our case and similarly to data emerging from the literature, $\mathrm{mRCC}$ is still challenging, not only because it represents the failure of early diagnosis, but also due to the lack of an established and well-defined management of complications. In conclusion, it can be considered a great imitator and a great dilemma for the clinicians.

\section{Learning points}

Diagnosis of mRCC is not simple, and abdominal imaging in patients with bone pain without evidence of skeleto-muscular origin should be considered. Nowadays, the management of this condition must be tailored on single patient, based on symptoms, comorbidity and IMDC risk score. Multidisciplinary approach and an immunotherapy-based combination therapy are the key points.

\section{Acknowledgments}

None to declare.

\section{Financial Disclosure}

None to declare.

\section{Conflict of Interest}

The authors do not have any conflict of interest.

\section{Informed Consent}

Written informed consent of patient was obtained.

\section{Author Contributions}

Conceptualization: Umberto Maestroni, Donatello Gasparro, and Davide Campobasso. Data curation: Giulio Guarino and Donatello Gasparro. Writing of original draft: Umberto Maestroni and Davide Campobasso. Writing of review and editing: Davide Campobasso and Francesco Ziglioli. All authors have read and agreed to the published version of the manuscript.

\section{Data Availability}

The authors declare that data supporting the findings of this study are available within the article.

\section{References}

1. Ferlay J, Colombet M, Soerjomataram I, Dyba T, Randi G, Bettio M, Gavin A, et al. Cancer incidence and mortality patterns in Europe: Estimates for 40 countries and 25 major cancers in 2018. Eur J Cancer. 2018;103:356-387.

2. Dudani S, de Velasco G, Wells JC, Gan CL, Donskov F, Porta C, Fraccon A, et al. Evaluation of clear cell, papillary, and chromophobe renal cell carcinoma metastasis sites and association with survival. JAMA Netw Open. 2021;4(1):e2021869.

3. Choueiri TK, Motzer RJ. Systemic therapy for metastatic renal-cell carcinoma. N Engl J Med. 2017;376(4):354366.

4. Roberto M, Botticelli A, Panebianco M, Aschelter AM, Gelibter A, Ciccarese C, Minelli M, et al. Metastatic renal cell carcinoma management: from molecular mechanism to clinical practice. Front Oncol. 2021;11:657639.

5. Tsili AC, Andriotis E, Gkeli MG, Krokidis M, Stasinopoulou M, Varkarakis IM, Moulopoulos LA, et al. The role of imaging in the management of renal masses. Eur J Radiol. 2021;141:109777.

6. Padala SA, Barsouk A, Thandra KC, Saginala K, Mohammed A, Vakiti A, Rawla P, et al. Epidemiology of renal cell carcinoma. World J Oncol. 2020;11(3):79-87.

7. Mazzaschi G, Quaini F, Bersanelli M, Buti S. Cytoreductive nephrectomy in the era of targeted- And immuno- 
therapy for metastatic renal cell carcinoma: An elusive issue? A systematic review of the literature. Crit Rev Oncol Hematol. 2021;160:103293.

8. Pagnini F, Cervi E, Maestroni U, Agostini A, Borgheresi A, Piacentino F, Angileri SA, et al. Imaging guided percutaneous renal biopsy: do it or not? Acta Biomed. 2020;91(8-S):81-88.

9. Galosi AB, Papaveri A, Castellani D, Agostini E, Burattini L, Dell'Atti L. Level IV tumor thrombus in nonmetastatic renal cell cancer? No, thanks. Level II is better. Lessons learned from a case report. Urol Case Rep. 2021;37:101660.

10. Buti S, Petrelli F, Ghidini A, Vavassori I, Maestroni U, Bersanelli M. Immunotherapy-based combinations versus standard first-line treatment for metastatic clear cell renal cell carcinoma: a systematic review and meta-analysis. Clin Transl Oncol. 2020;22(9):1657-1663.

11. Mir MC, Matin SF, Bex A, Spiess PE, Thompson RH, Grob B, van Poppel H. The role of surgery in the management of metastatic kidney cancer: an evidence-based collaborative review. Minerva Urol Nefrol. 2018;70(2):109125 .

12. Donini M, Buti S, Massari F, Mollica V, Rizzo A, Mon- tironi R, Bersanelli M, et al. Management of oligometastatic and oligoprogressive renal cell carcinoma: state of the art and future directions. Expert Rev Anticancer Ther. 2020;20(6):491-501.

13. Buti S, Bersanelli M, Viansone A, Leonetti A, Masini C, Ratta R, Procopio G, et al. Treatment Outcome of metastatic lesions from renal cell carcinoma underGoing Extra-cranial stereotactic body radioTHERapy: The together retrospective study. Cancer Treat Res Commun. 2020;22:100161.

14. Mosele GR, Caggiari G, Scarpa RM, Doria C. The treatment of vertebral metastases from renal cell carcinoma: a retrospective study. Minerva Urol Nefrol. 2017;69(2):166-172.

15. Bersanelli M, Buti S, Rizzo M. The need for new algorithms of treatment sequencing in clear-cell metastatic renal cell carcinoma. Expert Rev Anticancer Ther. 2021;21(4):401-412.

16. Bersanelli M, Gnetti L, Varotti E, Ampollini L, Carbognani P, Leonardi F, Rusca M, et al. Immune context characterization and heterogeneity in primary tumors and pulmonary metastases from renal cell carcinoma. Immunotherapy. 2019;11(1):21-35. 